\title{
Article \\ Development of Machine Learning Models for Prediction of Smoking Cessation Outcome
}

\author{
Cheng-Chien Lai ${ }^{1}{ }^{\mathbb{D}}$, Wei-Hsin Huang ${ }^{2}{ }^{(}$, Betty Chia-Chen Chang ${ }^{2}$ and Lee-Ching Hwang $2,3, * \mathbb{C}$ \\ 1 Department of Medical Education, Taipei Veterans General Hospital, Taipei City 11217, Taiwan; \\ kelvin2005s@gmail.com \\ 2 Department of Family Medicine, Mackay Memorial Hospital 25160, Taipei City 11217, Taiwan; \\ whh.5881@mmh.org.tw (W.-H.H.); betty905@gmail.com (B.C.-C.C.) \\ 3 Department of Medicine, Mackay Medical College, New Taipei City 25245, Taiwan \\ * Correspondence: hlc@mmh.org.tw
}

Citation: Lai, C.-C.; Huang, W.-H.; Chang, B.C.-C.; Hwang, L.-C. Development of Machine Learning Models for Prediction of Smoking Cessation Outcome. Int. J. Environ. Res. Public Health 2021, 18, 2584. https://doi.org/10.3390/ ijerph18052584

Academic Editor: Paul B. Tchounwou

Received: 24 January 2021

Accepted: 28 February 2021

Published: 5 March 2021

Publisher's Note: MDPI stays neutral with regard to jurisdictional claims in published maps and institutional affiliations.

Copyright: (c) 2021 by the authors. Licensee MDPI, Basel, Switzerland. This article is an open access article distributed under the terms and conditions of the Creative Commons Attribution (CC BY) license (https:// creativecommons.org/licenses/by/ $4.0 /)$

\begin{abstract}
Predictors for success in smoking cessation have been studied, but a prediction model capable of providing a success rate for each patient attempting to quit smoking is still lacking. The aim of this study is to develop prediction models using machine learning algorithms to predict the outcome of smoking cessation. Data was acquired from patients underwent smoking cessation program at one medical center in Northern Taiwan. A total of 4875 enrollments fulfilled our inclusion criteria. Models with artificial neural network (ANN), support vector machine (SVM), random forest (RF), logistic regression (LoR), k-nearest neighbor (KNN), classification and regression tree (CART), and naïve Bayes (NB) were trained to predict the final smoking status of the patients in a six-month period. Sensitivity, specificity, accuracy, and area under receiver operating characteristic (ROC) curve (AUC or ROC value) were used to determine the performance of the models. We adopted the ANN model which reached a slightly better performance, with a sensitivity of 0.704 , a specificity of 0.567 , an accuracy of 0.640 , and an ROC value of 0.660 (95\% confidence interval (CI): 0.617-0.702) for prediction in smoking cessation outcome. A predictive model for smoking cessation was constructed. The model could aid in providing the predicted success rate for all smokers. It also had the potential to achieve personalized and precision medicine for treatment of smoking cessation.
\end{abstract}

Keywords: smoking cessation; predictive model; machine learning; artificial neural network; precision medicine

\section{Introduction}

Cigarette smoking is an important global health issue [1] and is a well-known modifiable risk factor for many diseases including cancer, cardiovascular diseases, respiratory diseases, malfunction of the reproductive system, and many other organ systems [2]. It is estimated that smoking and exposure to secondhand smoke reduced life expectancy by 15 years [3]. Smoking cessation has been proven to be beneficial in many aspects of human health, including decreasing the risk of lung cancers, other cancers, heart attack, stroke, and chronic lung disease [4]. In addition, risk of all-cause mortality can be reduced by $13 \%$ within the first five years of smoking cessation [5]. More than one quarter of adult smokers are making attempts to quit smoking [6]. Assisting patients in quitting smoking is thus an important task for healthcare providers.

Evidence-based treatment and guideline for assistance in smoking cessation had been proposed and promoted [7]; however, only less than one third of the participants could achieve the goal of abstinence [8]. Many physicians found counseling for smoking cessation ineffective and time-consuming [9], and did not routinely do so in daily practice. To overcome this problem, several factors had been proposed to identify smokers who had a better chance of quitting, including the level of nicotine dependence, exhaled carbon monoxide (CO) concentration, cigarette amount per day, the age at smoking initiation, previous quit 
attempts, marital status, emotional distress, temperament and impulsivity scores, and the motivation to stop smoking [10-13]. However, individual use of these factors for prediction could lead to conflicting results that were not straightforward enough for the physicians and patients to interpret and apply. Providing a prediction model might be a favorable way to understand the chance of quitting smoking for each individual smoker.

Health outcome prediction models had been developed using methods of machine learning over recent years $[14,15]$. Some examples included prediction of postoperative inhospital mortality [16], complications in patients with diabetes mellitus [17], and occurrence of cardiovascular diseases in patients on dialysis [18]. For smoking cessation, a decision tree model developed with machine learning to predict smoking cessation treatment outcome was proposed by Coughlin et al in 2018 [19]. The study included 161 participants, with 90 in the training dataset and 71 in the validation dataset, yielding an average correct classification rate of about $64 \%$. However, a prediction model for smoking cessation constructed with a larger dataset is still lacking.

In this study, we aimed to construct a prediction model for smoking cessation using machine learning algorithms with a larger dataset to give more informative and more reliable results. Input features included parameters that could be readily collected at the first visit of the patient, so the model could be applied without difficulties by the physicians and the patients. The output of the model would be a prediction of success rate for smoking cessation. With a predicted success rate, shared decision making could be made more easily with patients who wished to quit smoking.

\section{Materials and Methods}

\subsection{Data Acquirement}

Data of patients enrolled in the smoking cessation program between 2010 and 2018 in a medical center in northern Taiwan was reviewed. A full course of the program lasted for 8 weeks, and each patient could enroll in the program at most twice a year, based on the regulations of Taiwan Health Promotion Administration. Each treatment course consisted of several visits to the outpatient clinics of a physician or a health educator. Exhaled CO level was obtained at each visit. Physicians would prescribe medication for patients based on clinical judgments. We inquired about the abstinence status of the patient 3 months and 6 months after the first visit by telephone. Each enrollment was viewed as a set of independent data. This study was approved by the Ethics Committee of Mackay Memorial Hospital (Institutional review board number: 17MMHIS049).

Out of the 7424 enrollments reviewed in the study, 2549 were excluded due to lack of data on abstinence status, other missing data, incorrectly recorded value, or a recorded body weight out of the desirable range of $30 \mathrm{~kg}$ to $150 \mathrm{~kg}$. The range was set to exclude extreme outliers that may decrease the discrimination ability of the specific feature in the model. The remaining 4875 enrollments were included for further analysis. The flowchart of data inclusion and preprocessing was shown in Figure 1.

\subsection{Feature Selection and Data Preprocessing}

Input features applied for the model included: sex, age, body weight, duration of smoking, daily amount of cigarette, motivation for smoke cessation, counseling done by health educator or physician, total score of the Fagerstrom Test for Nicotine Dependence (FTND) questionnaire [20], as well as the individual score of the 6 items in the questionnaire, conduction of $\mathrm{CO}$ test and the exhaled $\mathrm{CO}$ level, medication prescribed at first visit (no medication, nicotine replacement therapy (NRT), bupropion, or varenicline), and use of varenicline during the course. The 6 items in the FTND questionnaire included (1) Number of cigarettes smoked per day: 10 or less $=0 ; 11-20=1 ; 21-30=2 ; 31$ or more $=3$. (2) Time to first cigarette of the day: $60 \mathrm{~min}$ or more $=0 ; 31-60 \mathrm{~min}=1 ; 6-30 \mathrm{~min}=2 ; 0-5 \mathrm{~min}=3$. (3) Having difficulty not smoking in no-smoking areas: $\mathrm{No}=0$; Yes $=1$. (4) Which cigarette would be the most difficult to give up? First in the morning $=1$; Others $=0$. (5) Smoke 
more frequently in the first hours after waking: No $=0$; Yes $=1$. (6) Still smoke when ill in bed: No $=0$; Yes $=1$.

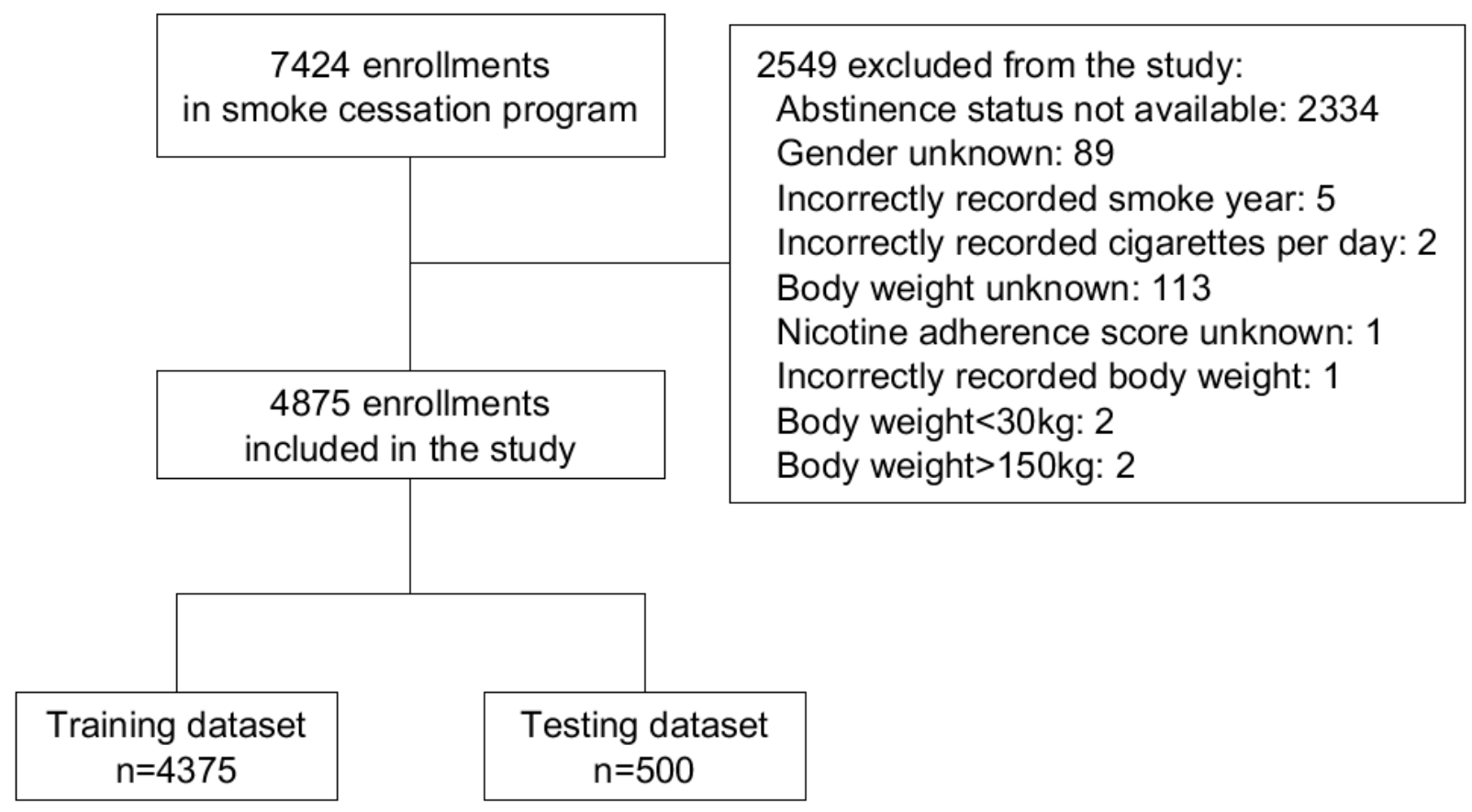

Figure 1. Flowchart of data inclusion and preprocessing.

We chose to include these predictors in our model based on that (1) It showed the patient's baseline characteristics, (2) It had been proven to be an independent predictor in previous studies, and (3) It was the treatment the patient received. It is likely that including well-proven predictors provides more information to the outcome, thus improves the model performance.

The primary outcome of the model was a binary variable $(1,0)$ defined as the final abstinence status available from the patient within 6 months. If a patient had reported the abstinence status at the time at 6-month follow up, the result of 6-month follow up would be used. On the other hand, if a patient had not reported the abstinence status at 6-month follow up but had reported at 3 month follow-up, the result of 3-month follow up would be used. If a patient did not answer both times, the data would be excluded. Out of the 4875 enrollments, $3680(75.5 \%)$ of the abstinence status was obtained from the 6-month period, and 1195 (24.5\%) was obtained from the 3-month period.

The data was randomly divided into 4375 enrollments in the training dataset and 500 enrollments in the testing dataset. We limited the testing dataset to 500 samples in order to reserve more samples to be used for model training, and also ensure that the number of testing data was adequate for statistical analysis. The 4375:500 split was close to 90:10, and it was common to apply a split of either 70:30, 80:20, or 90:10 for construction of machine learning models $[16,21,22]$. For the artificial neural network (ANN), support vector machine (SVM), and k-nearest neighbor $(\mathrm{KNN})$ models, the training data was rescaled to the range of 0 to 1 in every feature, and the testing data was rescaled according to the rescale index of the training data.

\subsection{Machine Learning Model Development}

In our study, we tried to develop a machine learning model to predict the probability of smoking cessation with features available at first visit. The candidate algorithms included: ANN, SVM, random forest (RF), logistic regression (LoR), KNN, classification and regression tree (CART), and naïve Bayes (NB). 
The ANN model was made with Python 3.7 (Python Software Foundation, Wilmington, DE, USA) using Tensorflow 1.14.0 (Google Brain Team, Mountain View, CA, USA). The ANN was designed as a feedforward network with 4 fully-connected layers: the input layer consisted of 20 nodes, the hidden layer one consisted of 10 nodes, the hidden layer two consisted of 5 nodes, and the output layer consisted of 2 nodes. One-hot-encoding was used for the output layer, so that the probability of each categorical output could be calculated. For each connection between layers, the weight function was initialized randomly with normal distribution, and the bias function was initialized with zeros. The loss function of the model was defined as cross entropy. During the training process of the model, the training dataset would be given to the ANN, and the ANN would learn by optimizing the weight and bias between the connections, in order to minimize the loss function, at a learning rate optimized with adaptive moment estimation. To avoid overfitting of the training dataset, early stopping and a dropout rate of 0.4 were applied.

For other methods, including SVM, RF, LoR, KNN, CART, and NB, the training process was achieved with Python 3.7 using Scikit-learn 0.21.2 [23]. For SVM, RF, KNN, and CART, a $20 \%$ validation dataset was derived randomly from the training dataset for each training section. Hyperparameters of the models were adjusted aggressively with experiments to achieve the best performance for the validation dataset. We prevented overfitting by limiting the complexities of the models. The final adopted SVM model was using linear classifier as kernel function; the RF model held 100 trees with maximum features and maximum depth set to 8; the KNN model was set to 81 neighbors. For LoR and NB, the whole training dataset was used due to the fixed results and the absence of adjustable hyperparameters.

\subsection{Statistical Analysis}

The testing dataset was used for further statistical analysis of the performance of the machine learning models. For each model, the result of the output was transferred to a number between 0 to 1 indicating the predicted success rate for smoking cessation. By design, the best performance of the models would be given at the cutoff point 0.5 , which meant that any output value greater than or equal to 0.5 would be considered a positive prediction (or abstinence in the study), and any output value less than 0.5 would be considered a negative prediction (or non-abstinence in the study). By adjusting the cutoff point, different combinations of sensitivity and specificity could be achieved. Hence, an ROC curve could be drawn by moving the cutoff point from 0 to 1 . The sensitivity, specificity, accuracy, and the ROC value were calculated to examine the performance of the machine learning models. The calculation of the CI for the ROC values and the comparison between different ROC values were performed with MedCalc 19.2 using the method proposed by DeLong et al [24].

\subsection{Application of the Machine Learning Model}

An application was made for physicians and patients to utilize the machine learning model. Through entering or changing the input values, the predicted success rate for smoking cessation would change accordingly.

\section{Results}

\subsection{Characteristics of Enrolled Data}

Of the 4875 enrollments including 3835 (78.7\%) men and $1040(21.3 \%)$ women, the average age was 46.7 years old and the average duration of smoking was 25.0 years. Over the eight-week period of the smoking cessation program, the average number of visits was $2.1 \pm 1.1$. The abstinence rate was $53.6 \%(n=2675)$. The 4875 enrollments were randomly split into 4375 in the training dataset and 500 in the testing dataset. The abstinence rate was $53.7 \%$ in the training dataset and $53.4 \%$ in the testing dataset $(p=0.9092)$. Comparison of characteristics between the training and testing dataset were shown in Table 1. The statistics for homogeneity testing were shown in Supplementary Table S1. 
Table 1. Baseline characteristics of the training and testing dataset.

\begin{tabular}{|c|c|c|c|c|c|}
\hline & & $\begin{array}{c}\text { Total } \\
(n=4875)\end{array}$ & $\begin{array}{l}\text { Training Dataset } \\
\quad(n=4375)\end{array}$ & $\begin{array}{l}\text { Testing Dataset } \\
\quad(n=500)\end{array}$ & $p$-Value \\
\hline \multirow[t]{2}{*}{ Gender $(n, \%)$} & Female & $1040(21.3)$ & $938(21.4)$ & $102(20.4)$ & 0.5907 \\
\hline & Male & $3835(78.7)$ & $3437(78.6)$ & $398(79.6)$ & \\
\hline Age (years) & & $46.7 \pm 12.7$ & $46.7 \pm 12.7$ & $46.9 \pm 13.0$ & 0.8325 \\
\hline Body weight $(\mathrm{kg})$ & & $71.0 \pm 14.9$ & $71.1 \pm 15.0$ & $70.6 \pm 14.3$ & 0.5200 \\
\hline Duration of smoking (years) & & $25.0 \pm 12.2$ & $25.1 \pm 12.2$ & $24.7 \pm 12.2$ & 0.5294 \\
\hline $\begin{array}{c}\text { Number of cigarettes smoked per day at } \\
\text { baseline (stick) }\end{array}$ & & $20.1 \pm 12.3$ & $20.1 \pm 12.4$ & $20.2 \pm 11.9$ & 0.8987 \\
\hline \multirow[t]{2}{*}{ Ambition (urge to quit) } & Yes & $2692(55.2)$ & $2430(55.5)$ & $262(52.4)$ & 0.1806 \\
\hline & No & $2183(44.8)$ & $1945(44.5)$ & $238(47.6)$ & \\
\hline \multirow[t]{2}{*}{ Physician clinics visit } & Yes & $4393(90.1)$ & $3937(90.0)$ & $456(91.2)$ & 0.3899 \\
\hline & No & $482(9.9)$ & $438(10.0)$ & $44(8.8)$ & \\
\hline \multirow[t]{2}{*}{ Educator clinics visit } & Yes & $1426(29.3)$ & $1289(29.5)$ & $137(27.4)$ & 0.3368 \\
\hline & No & 3449 (70.7) & $3086(70.5)$ & $363(72.6)$ & \\
\hline FTND score (point) & & $6.4 \pm 2.3$ & $6.4 \pm 2.3$ & $6.2 \pm 2.4$ & 0.1400 \\
\hline \multirow[t]{2}{*}{ Exhaled CO level tested at baseline } & Yes & $3987(81.8)$ & $3576(81.7)$ & $411(82.2)$ & 0.7995 \\
\hline & No & $888(18.2)$ & $799(18.3)$ & $89(17.8)$ & \\
\hline \multirow{5}{*}{$\begin{array}{l}\text { Exhaled CO levels (ppm) } \\
\text { Smoking cessation drugs prescribed at the } \\
\text { 1st visit }\end{array}$} & & $15.9 \pm 10.1$ & $15.9 \pm 10.1$ & $16.0 \pm 10.3$ & 0.7581 \\
\hline & Nil & $441(9.1)$ & $399(9.1)$ & $42(8.4)$ & 0.1406 \\
\hline & NRT & $943(19.3)$ & $846(19.3)$ & $97(19.4)$ & \\
\hline & Bupropion & $9(0.2)$ & $6(0.1)$ & $3(0.6)$ & \\
\hline & Varenicline & $3482(71.4)$ & $3124(71.4)$ & $358(71.6)$ & \\
\hline \multirow[t]{2}{*}{ Use varenicline during treatment } & No & $1338(27.4)$ & $1204(27.5)$ & $134(26.8)$ & 0.7325 \\
\hline & Yes & $3537(72.6)$ & $3171(72.5)$ & $366(73.2)$ & \\
\hline \multirow[t]{2}{*}{ Point prevalence abstinence $(n, \%)$} & Success & 2615(53.6) & 2348 (53.7) & $267(53.4)$ & 0.9092 \\
\hline & Fail & $2260(46.4)$ & 2027 (46.3) & $233(46.6)$ & \\
\hline
\end{tabular}

FTND: Fagerström Test of Nicotine Dependence; CO: Carbon monoxide; NRT: Nicotine Replacement Therapy.

\subsection{Model Performance}

After training the machine learning models with the training dataset, the testing dataset was used to test for the performance. The sensitivities, specificities, accuracies, and ROC values were shown in Table 2. To compare the ROC values of different models, the $p$ value between every 2 models was calculated and presented in Table 3 (presented with $p$ value comparing the 2 models labeled by the corresponding column and row).

Table 2. Performance of different machine learning models for prediction of smoking cessation outcome.

\begin{tabular}{ccccc}
\hline & Sensitivity & Specificity & Accuracy & ROC Value (95\% CI) \\
\hline ANN & 0.704 & 0.567 & 0.640 & $0.660(0.617-0.702)$ \\
SVM & 0.768 & 0.433 & 0.612 & $0.658(0.614-0.699)$ \\
RF & 0.757 & 0.485 & 0.626 & $0.654(0.610-0.695)$ \\
LoR & 0.742 & 0.459 & 0.608 & $0.653(0.609-0.694)$ \\
KNN & 0.764 & 0.408 & 0.598 & $0.618(0.573-0.660)$ \\
CART & 0.674 & 0.528 & 0.606 & $0.612(0.568-0.655)$ \\
NB & 0.614 & 0.524 & 0.568 & $0.608(0.564-0.651)$
\end{tabular}

ANN: Artificial neural network; SVM: Support vector machine; RF: Random forest; LoR: Logistic regression; KNN: K-nearest neighbors; CART: Classification and regression tree; NB: Naïve Bayes; ROC value: Receiver operating characteristic value; CI: Confidence interval.

By observing the ROC values of the models, there was a trend that the ANN, SVM, $\mathrm{RF}$, and the LoR models had a better performance compared with the KNN, CART, and the NB models, though many of the comparisons were not statistically significant. Of the four better models, the ANN model had the best accuracy of 0.640 and an ROC value of 0.660 (95\% CI: $0.617-0.702$ ). The sensitivity and specificity of the ANN model was 0.704 and 0.567 respectively. As a result, the ANN model was adopted as the desired model for smoking cessation clinic setting in the study. The ROC curves of different models were 
shown in Figure 2, and the ROC curve of the ANN model with 95\% CI boundary was shown in Figure 3.

Table 3. Comparison of ROC values between different machine learning models for smoking cessation.

\begin{tabular}{cccccccc}
\hline & ANN & SVM & RF & LoR & KNN & CART & NB \\
\hline ANN & 1.0000 & & & & & & \\
SVM & 0.7997 & 1.0000 & & & & & \\
RF & 0.6882 & 0.8158 & 1.0000 & & & & \\
LoR & 0.4873 & 0.2595 & 0.9518 & 1.0000 & & & \\
KNN & 0.0491 & 0.0601 & 0.1308 & 0.0945 & 1.0000 & & \\
CART & 0.0505 & 0.058 & 0.0615 & 0.0944 & 0.8391 & 1.0000 & \\
NB & 0.0068 & 0.0009 & 0.0335 & 0.0031 & 0.6769 & 0.8865 & 1.0000 \\
\hline
\end{tabular}

The results were shown in $p$ value. ANN: Artificial neural network; SVM: Support vector machine; RF: Random forest; LoR: Logistic regression; KNN: K-nearest neighbors; CART: Classification and regression tree; NB: Naïve Bayes.

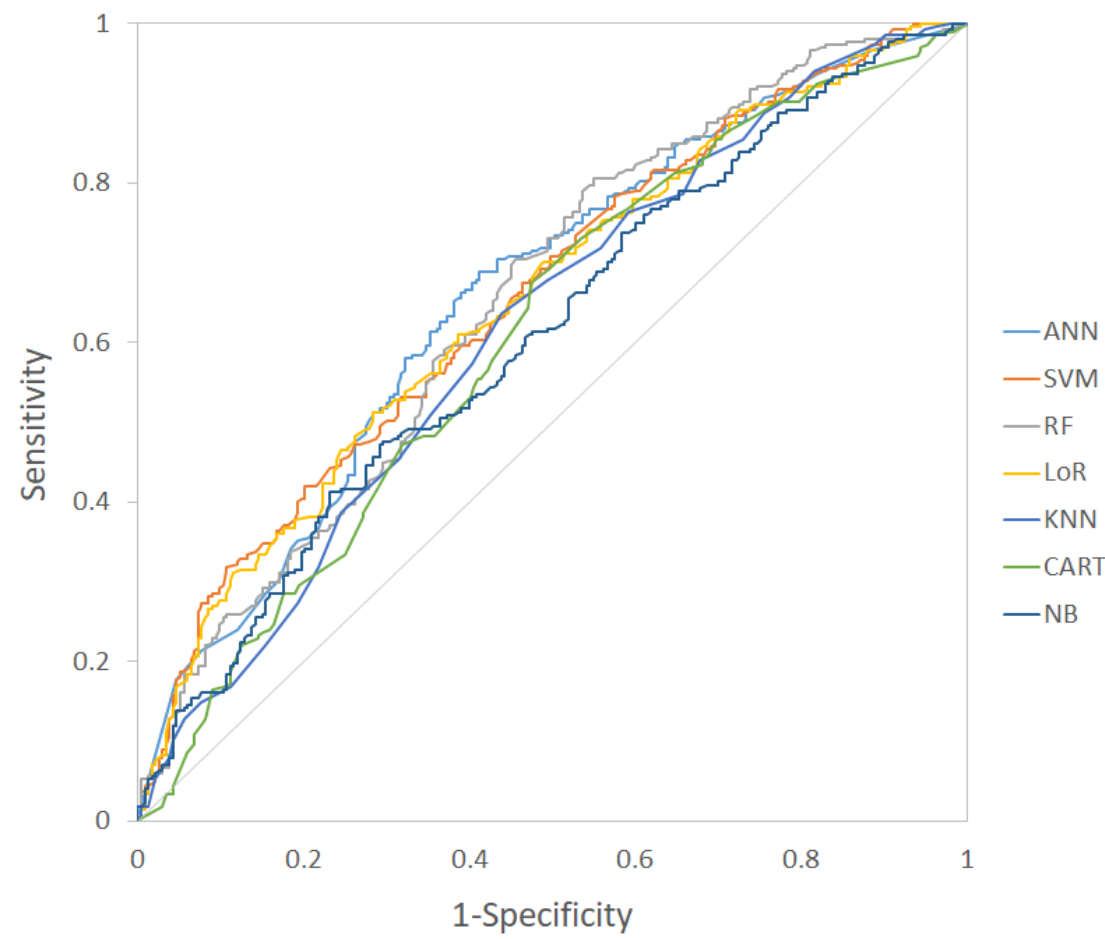

Figure 2. The ROC curves of different machine learning models for smoking cessation. ANN: Artificial neural network; SVM: Support vector machine; RF: Random forest; LoR: Logistic regression; KNN: K-nearest neighbors; CART: Classification and regression tree; NB: Naïve Bayes; ROC curve: Receiver operating characteristic curve. 


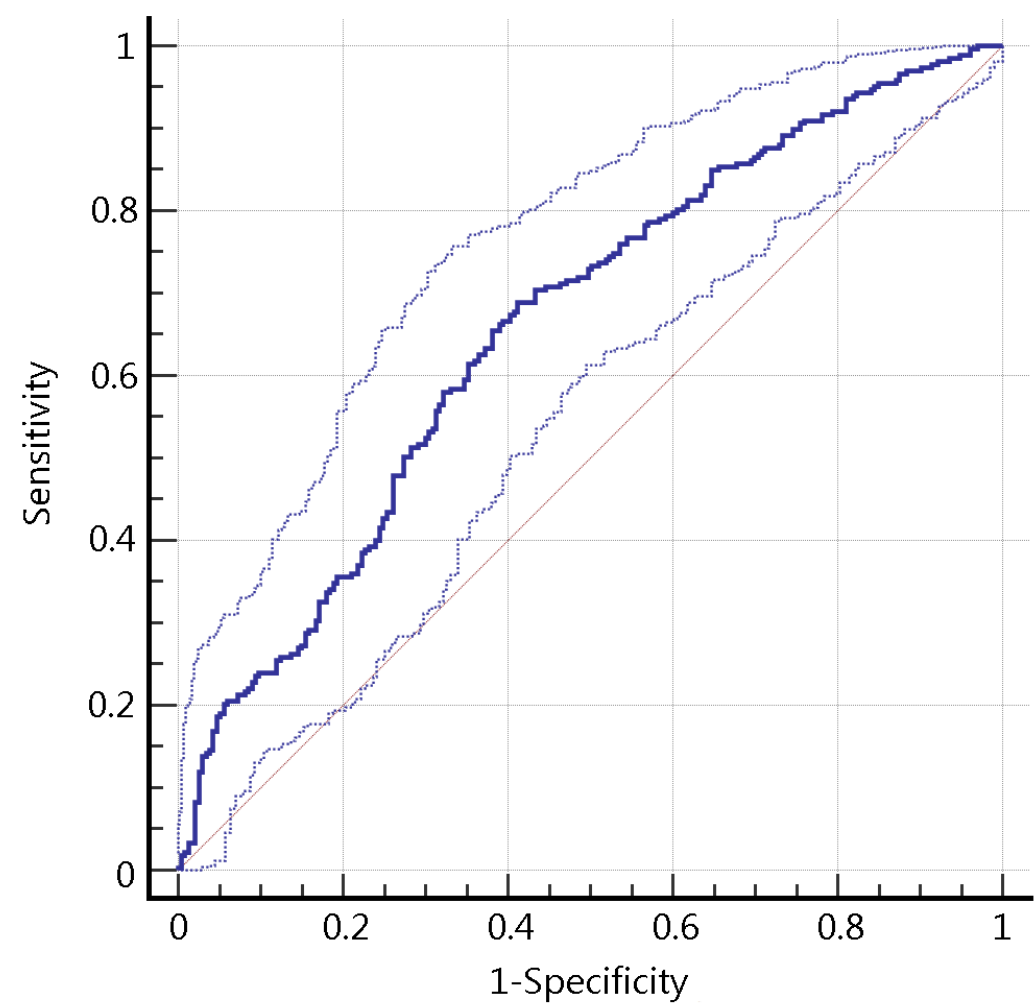

Figure 3. The ROC curve of the artificial neural network model, with $95 \%$ CI boundaries. ROC curve: Receiver operating characteristic curve; CI: Confidence interval.

\section{Discussion}

In our study, we constructed predictive models using seven different machine learning methods to predict the success rate of smoking cessation for current smokers with data available at the first visit. While the comparison of ROC between different models mostly revealed no statistical significance, the ANN model yield a better accuracy and ROC value, and was thus adopted as the desired model in the study.

Previous works on this topic focused mainly on identifying the independent predictors and describing the odds ratio for each predictor [11]. However, patients might host varying combinations of these predictors, which would lead to conflicting results and difficult interpretation for each individual in clinical practice. This situation could be avoided with the aid of prediction models. An attempt of constructing a classification model with machine learning on this topic was done by Coughlin et al. [19] in 2018. A decision tree model was made with an average correct classification rate of $64 \%$ of the full tree and $74 \%$ of the first split of tree in the validation cohort, and $81 \%$ of the full tree and $70 \%$ of the first split of tree in the training cohort, using delay discounting as the first split of the tree. The results were inspiring but should be interpreted carefully that overfitting might occur in a relatively small database, and the same protocol should be used in both the training and the validation cohort. In our study, a larger database $(n=4875)$ was used, and several different machine learning algorithms were applied. With the algorithms of machine learning, especially ANN, more flexible modeling could be achieved, with more complex pattern between the inputs and outputs identified [25].

The performance of our ANN model reached an AUC of 0.660, with a sensitivity of 0.704 and a specificity of 0.567 . While an AUC $>0.7$ was more preferable when analyzing examination tools [26], more work would be needed to achieve better AUC on predicting smoking cessation, including adding more features to the input. In our study, the 20 input features mainly consisted of the patient's physical status, smoking status, and the intervention provided. These features were chosen in consideration of their easy accessibility, and most of these features were previously proven to be independent predictors in smoking 
cessation. However, these information may contribute only to a limited portion of the final abstinence status of the patient. Other factors might also be important, including the patient's socioeconomic status, education level, family support, specific motivation for quitting, previous quit attempts, marital status, emotional distress, and the executive function and impulsivity measures. Addition of more related factors and recruitment of more data for training would be possible ways to further improve the performance of the model. However, adding too much input features also increases the barrier of applying the model in a clinical setting. The balance between model performance and convenience of use should be considered. Inter-correlation between selected features is a concern when more features are included. A correlation matrix of the input features in our study was provided in Supplementary Table S2. In this study, we considered 20 input features not being very much compared with previous studies $[14,16,18]$, and the algorithms constructed in our study were not very complex. Since the models were relatively simple, the influence of inter-correlation from data should be conquered during model training, and would not cast a major problem to the performance.

An advantage of using the model is to understand the individual impact of change for a certain factor on smoking cessation. For example, losing $5 \mathrm{~kg}$ of weight would increase the probability of quitting by $10 \%$ in one patient, while for another patient, losing weight might not have the same effect, but changing the medication from NRT to varenicline would increase that patient's probability of quitting by $15 \%$. This example demonstrates that good prediction models provide keys to personalized and precision medicine. This would also encourage the physician to find better ways of quitting for the patients, thereby increasing the patient's confidence, which is also an important component in successful smoking cessation. Further studies would be needed to confirm the effect of our prediction model in the clinical setting.

There were some limitations in the study. First, the outcome, the last available abstinence status within six months, was obtained through self-report by the patient. This could cause bias and misclassification. For an objective outcome measurement, exhaled $\mathrm{CO}[27,28]$ and urine cotinine level [29] could be used. In addition, $24.5 \%$ of the abstinence status was obtained from the three-month period, and $75.5 \%$ was from the six-month period. This time gap could cause bias for those who changed in abstinence status between the third and sixth month. To examine the effect of combining the three-month and six-month outcome to the prediction model, we rechecked the cessation rate in the original three-month and six-month report. The cessation rate was $55.6 \%$ at three month and $55.7 \%$ at six month. $p$-value of two-sample $\mathrm{z}$ test for cessation rate was 0.8887 . Due to the similarity in cessation rate, the influence of adopting the combined outcome should be negligible.

Second, each enrollment was considered independent in our study. Repeated enrollments might cause bias in model construction and validation. To estimate the extent of influence of repeated enrollments in our study, we re-examined the 4875 data to look for data pairs with strong similarity. We tested for data pairs (calculated 11,880,375 pairs) that satisfied the following criteria: (1) age difference $< \pm 3$ years, (2) sex was the same, (3) body weight difference $< \pm 2.5 \%$, (4) ambition was the same, (5) duration of smoking $< \pm 3$ years, (6) cigarettes per day $< \pm 10$ sticks, (7) total FTND score $< \pm 3$ points, and (8) outcome was the same. Of the 11,880,375 pairs of data, 1781 pairs were found to be "similar" according to this criteria, which consisted of about $0.015 \%$. With this level of similarity, the extent of bias caused by repeated enrollments might not be a major concern.

Third, other known and unknown predictors of smoking cessation might have been left out from our input features. However, if more features were to be included, the complexity of applying the model would become a barrier for clinical use. Furthermore, misreporting might occur during data collection of the input features, and we did not measure the compliance of the patients prescribed with medication. Lastly, the outcome of smoking cessation was related with race, culture, and health care policy. The model 
was based on medical practice in Taiwan and might not be able to be generalized to other countries worldwide.

\section{Conclusions}

A predictive model with ANN was constructed to predict the success rate of smoking cessation for current smokers using data available at the first visit. This model was easily applicable requiring only data collected at first patient visit. A predicted success rate could be provided for each patient, which could aid in shared decision making with the patient. Moreover, this method also had the potential to achieve personalized and precision medicine for treatment of smoking cessation.

Supplementary Materials: The following are available online at https:/ / www.mdpi.com/1660-4 601/18/5/2584/s1, Table S1: Homogeneity testing between the training and test groups, Table S2: Pearson's correlation coefficient matrix of predictors.

Author Contributions: Conceptualization, C.-C.L. and L.-C.H.; methodology, C.-C.L.; software, C.-C.L. and L.-C.H.; Validation, C.-C.L., W.-H.H., B.C.-C.C., and L.-C.H.; formal analysis, C.-C.L. and L.-C.H.; investigation, C.-C.L.; resources, W.-H.H. and L.-C.H.; data curation, C.-C.L. and L.-C.H.; writing—original draft preparation, C.-C.L.; writing—review and editing, B.C.-C.C. and L.-C.H.; visualization, C.-C.L.; supervision, L.-C.H.; project administration, L.-C.H. All authors have read and agreed to the published version of the manuscript.

Funding: This research received no external funding.

Institutional Review Board Statement: The study was conducted according to the guidelines of the Declaration of Helsinki, and approved by the Ethics Committee of Mackay Memorial Hospital (Institutional review board number: 17MMHIS049).

Informed Consent Statement: The data were analyzed anonymously and consent was not obtained from the participants.

Data Availability Statement: Data were available from the Outpatient Smoking Cessation Treatment Database established by Taiwan Health Promotion Administration. Due to legal restrictions imposed by the government of Taiwan in relation to the "Personal Information Protection Act", data cannot be made publicly available.

Acknowledgments: Our thanks to Taiwan Health Promotion Administration for advocating the tobacco hazards prevention and supporting smoking cessation services.

Conflicts of Interest: The authors declare no conflict of interest.

\section{References}

1. Peacock, A.; Leung, J.; Larney, S.; Colledge, S.; Hickman, M.; Rehm, J.; Giovino, G.A.; West, R.; Hall, W.; Griffiths, P. Global statistics on alcohol, tobacco and illicit drug use: 2017 status report. Addiction 2018, 113, 1905-1926. [CrossRef] [PubMed]

2. Onor, I.O.; Stirling, D.L.; Williams, S.R.; Bediako, D.; Borghol, A.; Harris, M.B.; Darensburg, T.B.; Clay, S.D.; Okpechi, S.C.; Sarpong, D.F. Clinical effects of cigarette smoking: Epidemiologic impact and review of pharmacotherapy options. Int. J. Environ. Res. Public Health 2017, 14, 1147. [CrossRef] [PubMed]

3. Unified Development Ordinance. The Health Consequences of Smoking - 50 Years of Progress: A Report of the Surgeon General; US Department of Health and Human Services: Atlanta, GA, USA, 2014.

4. Novello, A.C. Surgeon General's report on the health benefits of smoking cessation. Public Health Rep. 1990, 105, 545. [PubMed]

5. Kenfield, S.A.; Stampfer, M.J.; Rosner, B.A.; Colditz, G.A. Smoking and smoking cessation in relation to mortality in women. JAMA 2008, 299, 2037-2047. [CrossRef]

6. Goren, A.; Annunziata, K.; Schnoll, R.A.; Suaya, J.A. Smoking cessation and attempted cessation among adults in the United States. PLoS ONE 2014, 9, e93014. [CrossRef]

7. $\quad$ Fiore, M.C.; Jaén, C.R.; Baker, T.B.; Bailey, W.C.; Benowitz, N.L.; Curry, S.J;; Dorfman, S.F.; Froelicher, E.S.; Goldstein, M.G.; Healton, C.G. Treating Tobacco Use and Dependence: 2008 Update; US Department of Health and Human Services: Rockville, MD, USA, 2008.

8. Anthenelli, R.M.; Benowitz, N.L.; West, R.; St Aubin, L.; McRae, T.; Lawrence, D.; Ascher, J.; Russ, C.; Krishen, A.; Evins, A.E. Neuropsychiatric safety and efficacy of varenicline, bupropion, and nicotine patch in smokers with and without psychiatric disorders (EAGLES): A double-blind, randomised, placebo-controlled clinical trial. Lancet 2016, 387, 2507-2520. [CrossRef] 
9. Vogt, F.; Hall, S.; Marteau, T.M. General practitioners' and family physicians' negative beliefs and attitudes towards discussing smoking cessation with patients: A systematic review. Addiction 2005, 100, 1423-1431. [CrossRef]

10. Huang, W.-H.; Hsu, H.-Y.; Chang, B.C.-C.; Chang, F.-C. Factors correlated with success rate of outpatient smoking cessation services in Taiwan. Int. J. Environ. Res. Public Health 2018, 15, 1218. [CrossRef]

11. Caponnetto, P.; Polosa, R. Common predictors of smoking cessation in clinical practice. Respir. Med. 2008, 102, 1182-1192 [CrossRef] [PubMed]

12. Vangeli, E.; Stapleton, J.; Smit, E.S.; Borland, R.; West, R. Predictors of attempts to stop smoking and their success in adult general population samples: A systematic review. Addiction 2011, 106, 2110-2121. [CrossRef] [PubMed]

13. López-Torrecillas, F.; Perales, J.C.; Nieto-Ruiz, A.; Verdejo-García, A. Temperament and impulsivity predictors of smoking cessation outcomes. PLoS ONE 2014, 9, e112440. [CrossRef]

14. Krittanawong, C.; Zhang, H.; Wang, Z.; Aydar, M.; Kitai, T. Artificial intelligence in precision cardiovascular medicine. J. Am. Coll. Cardiol. 2017, 69, 2657-2664. [CrossRef]

15. Adam, N.R.; Wieder, R.; Ghosh, D. Data science, learning, and applications to biomedical and health sciences. Ann. N. Y. Acad. Sci. 2017, 1387, 5-11. [CrossRef]

16. Lee, C.K.; Hofer, I.; Gabel, E.; Baldi, P.; Cannesson, M. Development and validation of a deep neural network model for prediction of postoperative in-hospital mortality. Anesthesiology 2018, 129, 649. [CrossRef]

17. Rodriguez-Romero, V.; Bergstrom, R.F.; Decker, B.S.; Lahu, G.; Vakilynejad, M.; Bies, R.R. Prediction of Nephropathy in Type 2 Diabetes: An Analysis of the ACCORD Trial Applying Machine Learning Techniques. Clin. Transl. Sci. 2019, 12, 519-528. [CrossRef] [PubMed]

18. Mezzatesta, S.; Torino, C.; De Meo, P.; Fiumara, G.; Vilasi, A. A machine learning-based approach for predicting the outbreak of cardiovascular diseases in patients on dialysis. Comput. Methods Programs Biomed. 2019, 177, 9-15. [CrossRef] [PubMed]

19. Coughlin, L.N.; Tegge, A.N.; Sheffer, C.E.; Bickel, W.K. A machine-learning approach to predicting smoking cessation treatment outcomes. Nicotine Tob. Res. 2020, 22, 415-422. [CrossRef] [PubMed]

20. Heatherton, T.F.; Kozlowski, L.T.; Frecker, R.C.; FAGERSTROM, K.O. The Fagerström test for nicotine dependence: A revision of the Fagerstrom Tolerance Questionnaire. Br. J. Addict. 1991, 86, 1119-1127. [CrossRef]

21. Kim, J.S.; Merrill, R.K.; Arvind, V.; Kaji, D.; Pasik, S.D.; Nwachukwu, C.C.; Vargas, L.; Osman, N.S.; Oermann, E.K.; Caridi, J.M.; et al. Examining the Ability of Artificial Neural Networks Machine Learning Models to Accurately Predict Complications Following Posterior Lumbar Spine Fusion. Spine 2018, 43, 853-860. [CrossRef] [PubMed]

22. Hamori, S.; Kawai, M.; Kume, T.; Murakami, Y.; Watanabe, C. Ensemble learning or deep learning? Application to default risk analysis. J. Risk Financ. Manag. 2018, 11, 12. [CrossRef]

23. Pedregosa, F.; Varoquaux, G.; Gramfort, A.; Michel, V.; Thirion, B.; Grisel, O. Scikit-learn: Machine Learning in Python. J. Mach. Learn. Res. 2012, 12, 2825-2830.

24. DeLong, E.R.; DeLong, D.M.; Clarke-Pearson, D.L. Comparing the areas under two or more correlated receiver operating characteristic curves: A nonparametric approach. Biometrics 1988, 44, 837-845. [CrossRef] [PubMed]

25. Dreiseitl, S.; Ohno-Machado, L. Logistic regression and artificial neural network classification models: A methodology review. J. Biomed. Inform. 2002, 35, 352-359. [CrossRef]

26. Witten, I.H.; Frank, E.; Hall, M.A. Chapter 5-Credibility: Evaluating What's Been Learned. In Data Mining: Practical Machine Learning Tools and Techniques, 3rd ed.; Witten, I.H., Frank, E., Hall, M.A., Eds.; Morgan Kaufmann: Boston, MA, USA, 2011; pp. 147-187. [CrossRef]

27. SRNT Subcommittee on Biochemical Verification. Biochemical verification of tobacco use and cessation. Nicotine Tob. Res. 2002, 4, 149-159. [CrossRef]

28. Benowitz, N.L.; Bernert, J.T.; Foulds, J.; Hecht, S.S.; Jacob, P.; Jarvis, M.J.; Joseph, A.; Oncken, C.; Piper, M.E. Biochemical Verification of Tobacco Use and Abstinence: 2019 Update. Nicotine Tob. Res. 2019. [CrossRef] [PubMed]

29. Hukkanen, J.; Jacob, P.; Benowitz, N.L. Metabolism and disposition kinetics of nicotine. Pharmacol. Rev. 2005, 57, 79-115. [CrossRef] [PubMed] 